\title{
SIRT4 suppresses the PI3K/Akt/NF-кB signaling pathway and attenuates HUVEC injury induced by oxLDL
}

\author{
YU TAO $^{1 *}$, SONGPING YU $^{1 *}$, MIN CHAO $^{2}$, YANG WANG $^{3}$, JIANHUA XIONG $^{4}$ and HENGLI LAI ${ }^{1}$ \\ ${ }^{1}$ Department of Cardiology, Jiangxi Provincial People's Hospital, Nanchang, Jiangxi 330006; ${ }^{2}$ Department of \\ Internal Medicine, Shanggao Hospital of Traditional Chinese Medicine, Yichun, Jiangxi 336400; ${ }^{3}$ Dexing Hospital of \\ Traditional Chinese Medicine, Dexing, Jiangxi 334200; ${ }^{4}$ Department of Geriatrics, \\ Fuzhou First People's Hospital, Fuzhou, Jiangxi 344000, P.R. China
}

Received July 10, 2018; Accepted March 27, 2019

DOI: $10.3892 / \mathrm{mmr} .2019 .10161$

\begin{abstract}
Atherosclerosis is a chronic and progressive disease. Its morbidity and mortality rates have demonstrated an increase in recent years. The present study aimed to explore the role of sirtuin (SIRT) 4 in the development of atherosclerosis. Alterations in SIRT4 expression in response to oxidized low density lipoprotein (oxLDL) were quantified in human umbilical vein endothelial cells (HUVECs) using western blotting. Cell counting kit- 8 and flow cytometry assays were used in order to explore the effects of SIRT4 on HUVEC proliferation and apoptosis. The effect of SIRT4 on the expression of inflammatory factors in HUVECs was analyzed using ELISA. The expression and phosphorylation of proteins in the phosphoinositide 3-kinase (PI3K)/protein kinase B (Akt)/nuclear factor (NF)- $\kappa \mathrm{B}$ pathway were comparatively analyzed using western blotting. Nuclear translocation of p $65 \mathrm{NF}-\kappa \mathrm{B}$ was examined using immunofluorescence. The present study indicated that oxLDL treatment decreased the expression of SIRT4 in HUVECs in a dose- and time-dependent manner. SIRT4 overexpression promoted oxLDL-induced HUVEC proliferation and inhibited cell apoptosis. Furthermore, SIRT4 overexpression suppressed the PI3K/Akt/NF- $\mathrm{BB}$ pathway by inhibiting PI3K phosphorylation and phosphorylated (p)-Akt, p-nuclear factor of kappa light polypeptide gene enhancer in B-cells inhibitor $\alpha$ and p-p65 NF- $\mathrm{BB}$ expression; blocking p65 NF- $\kappa \mathrm{B}$ nuclear translocation and decreasing interleukin (IL)-1 $\beta$, IL-6, and tumor necrosis factor $\alpha$ expression in oxLDL-induced HUVECs. In conclusion, SIRT4 overexpression enhanced HUVEC survival, suppressed the
\end{abstract}

Correspondence to: Professor Hengli Lai, Department of Cardiology, Jiangxi Provincial People's Hospital, 92 Aiguo Road, Donghu, Nanchang, Jiangxi 330006, P.R. China

E-mail: laihengli1972@163.com

${ }^{*}$ Contributed equally

Key words: sirtuin 4, atherosclerosis, inflammatory cytokine, nuclear factor- $\kappa \mathrm{B}$
$\mathrm{PI} 3 \mathrm{~K} / \mathrm{Akt} / \mathrm{NF}-\mathrm{\kappa B}$ signaling pathway and inhibited the expression of inflammatory cytokines in oxLDL-induced HUVECs.

\section{Introduction}

Atherosclerosis is a major disease that has a severe effect on human health. Its morbidity and mortality rates have demonstrated an increase in recent years $(1,2)$. Atherosclerosis develops due to impairment of molecular and cellular activities, leading to the disruption of vascular homeostasis (3). Currently, this disease is primarily considered as a form of chronic inflammation, which arises in response to lipid accumulation. Macrophages are effector cells which stimulate vascular inflammatory reactions throughout the pathological process (4). An increasing number of factors reportedly participate in the inflammatory response in atherosclerosis $(5,6)$; however, the detailed mechanisms underlying this process remain to be elucidated. Thus, an improved understanding of the pathophysiology of atherosclerosis in addition to the development of effective treatment methods are of great importance.

Sirtuin (SIRT) 4 is a member of the sirtuin protein family and is abundant in the heart, brain, kidney, liver and skeletal muscles (7). The Sirtuin family of proteins, from SIRT1 to SIRT7, serve key roles in the prevention of atherosclerosis (8). SIRT1 and SIRT6 inhibit foam cell formation and promote foam cell egress to prevent atherogenesis $(9,10)$. SIRT2 may regulate macrophage polarization in order to inhibit atherosclerotic plaque progression (11). SIRT4, initially reported as not showing nicotinamide adenine dinucleotide (NAD)-dependent deacetylase activity (12), is localized in the mitochondrial matrix (13). It uses NAD to adenosine diphosphate ribosylate glutamate dehydrogenase $(\mathrm{GDH})$ and suppresses GDH activity, limiting the generation of adenosine triphosphate (14). A previous study suggested that SIRT4 is a major regulator of lipid metabolism (15). Under nutrient-replete conditions, SIRT4 acts to repress fatty acid oxidation and promote lipid anabolism (16). In a previous study, it was demonstrated that lipopolysaccharide (LPS) treatment significantly decreased the expression of SIRT4 at mRNA and protein levels in a dose-dependent manner (17). In colorectal cancer, SIRT4 suppressed the proliferation, migration and invasion of cancer 
cells through the inhibition of glutamine metabolism via upregulation of E-cadherin expression (18). SIRT4 overexpression protected against diabetic nephropathy by preventing glucose-induced podocyte apoptosis and production of reactive oxygen species (19). Our previous study indicated that SIRT4 may inhibit inflammatory responses in human umbilical vein endothelial cells (HUVECs) (20).

Oxidized low density lipoprotein (oxLDL) reportedly induces atherosclerosis by triggering endothelial cell damage, and promoting lipid accumulation and proinflammatory responses (21). Nuclear factor (NF)- $\kappa B$, a multifunctional transcription regulator, serves an important role in the inflammatory pathways of atherosclerosis, where expression of NF- $\kappa \mathrm{B}$ and its downstream genes can be regulated via the phosphoinositide 3-kinase (PI3K)/protein kinase B (Akt) signaling pathway, which serves a vital role in the processes of cell proliferation, apoptosis and inflammation $(22,23)$. The aim of the present study was to investigate the role of SIRT4 in the development of atherosclerosis. The results indicated that oxLDL reduced the expression of SIRT4 in HUVECs. In addition, it was identified that overexpression of SIRT4 may reverse oxLDL-induced cell proliferation inhibition, rescue oxLDL-induced apoptosis and attenuate the expression of pro-inflammatory cytokines interleukin (IL)-1 $\beta$, IL-6 and tumor necrosis factor (TNF)- $\alpha$ induced by oxLDL, possibly via inhibition of the PI3K/Akt/NF-kB signaling pathway.

\section{Materials and methods}

Cell culture and treatment. HUVECs were obtained from Capsugel, Morristown, NJ, USA). The cells were cultured in Endothelial Growth Basal Medium (EBM-2; Lonza Group, Ltd., Basel, Switzerland) supplemented with growth factors according to the manufacturer's protocols. To analyze the changes in SIRT4 expression in response to oxLDL (Thermo Fisher Scientific, Inc., Waltham, MA, USA) at various concentrations, cells were treated with oxLDL for $24 \mathrm{~h}$ at concentrations of $0,10,50$ and $100 \mu \mathrm{M}$ at $37^{\circ} \mathrm{C}$. In order to analyze changes in SIRT4 expression in response to oxLDL treatment following different time periods, cells were treated with $50 \mu \mathrm{M}$ oxLDL for $0,12,24$ and $48 \mathrm{~h}$ at $37^{\circ} \mathrm{C}$. Following treatment, cells were harvested and assessed for changes in SIRT4 expression using western blotting.

Stable overexpression of SIRT4 and treatment. The complete open reading frame of SIRT4 was cloned into the pLVX-IRES-ZsGreen1 (Clontech Laboratories, Inc., Mountain View, CA, USA) plasmid (OV-SIRT4), using the empty vector as negative control (-NC). pLVX-SIRT4 was generated by transiently transfecting 293T cells (Beijing ZhongyuanLtd.,Beijing, China). Lentiviral production, concentration and titration were performed as follows: 293T cells (70-80\% confluence) were seeded in 6-well plates, and $1.5 \mu \mathrm{g}$ pLVX-IRES-ZsGreen1 was transfected using the Lipofectamine ${ }^{\circledR} 2000$ (40 $\mu$ l; Invitrogen; Thermo Fisher Scientific, Inc.) according to the manufacturer's protocol. Then, 293T cells were cultured for $48 \mathrm{~h}$ at $37^{\circ} \mathrm{C}$. Lentivirus particles were directly collected and concentrated from the cell culture medium at $48 \mathrm{~h}$ following transduction by multi-steps of ultracentrifugation $\left(50,000 \mathrm{x} \mathrm{g}\right.$ at $4^{\circ} \mathrm{C}$ for $2 \mathrm{~h}$ ). The titration (transduction units, TU) and multiplicity of infection of concentrated lentivirus particles were determined in $293 \mathrm{~T}$ cells grown in 96 -well plates by serial dilutions. For infection purposes, $2 \times 10^{5}$ HUVECs were divided into 2 groups and subcultured in 6-well culture plates for $24 \mathrm{~h}$ prior to transduction. For infection, the cell culture medium was removed and cells were washed twice with phosphate-buffered saline (PBS). Next, $0.5 \mathrm{ml}$ of lentiviral suspension $\left(1 \times 10^{8} \mathrm{IU} / \mathrm{ml}\right.$, multiplicity of infection=100) containing $8 \mu \mathrm{g} / \mathrm{ml}$ Polybrene was added to the cells. Cells were then incubated at $37^{\circ} \mathrm{C}$ overnight. Vector suspension was then aspirated from the cells and the transduced cells were added to $2 \mathrm{ml} /$ flask of fresh growth medium. Cells were then incubated at $37^{\circ} \mathrm{C}$ in a humidified atmosphere containing $5 \% \mathrm{CO}_{2}$. The growth medium was replaced after $24 \mathrm{~h}$. After allowing the cells to incubate for $72 \mathrm{~h}$ at $37^{\circ} \mathrm{C}$, HUVECs cells were passaged twice per week with growth medium containing $5 \mu \mathrm{g} / \mathrm{ml}$ puromycin to select for cells expressing the transduced vector. Positively screened cell lines were sub-cloned three times using limiting dilution (diluent: EBM-2) and cultured in growth medium containing puromycin for 1 month to generate stable cell lines. HUVECs were infected by pLVX-SIRT4 and treated with $50 \mu \mathrm{M}$ oxLDL. Following incubation for $48 \mathrm{~h}$ at $37^{\circ} \mathrm{C}$ and $5 \% \mathrm{CO}_{2}$, HUVECs overexpressing SIRT4 and NC cells were harvested for western blotting.

Western blotting. Following treatment or infection, cells were lysed using a cell lysis buffer containing protease inhibitors [Tris- $\mathrm{HCl}(\mathrm{pH} 7.5) 50 \mathrm{mM}, \mathrm{NaCl} 250 \mathrm{mM}$, EDTA $10 \mathrm{mM}$, NP-40 0.5\%, Leupeptin $10 \mu \mathrm{M}$, PMSF $1 \mathrm{mM}$ and NaF $4 \mathrm{mM}$ ]. The protein concentration was measured using a bicinchoninic acid protein assay kit (Thermo Fisher Scientific, Inc.). Soluble lysate was mixed with loading buffer and boiled for $5 \mathrm{~min}$. Equal amounts of the protein samples (50 $\mathrm{ng} / \mathrm{lane}$ ) were separated via $10 \%$ SDS-PAGE and transferred on to polyvinylidene difluoride membranes. Membranes were blocked with PBS, containing $10 \%$ non-fat dry milk, overnight at $4^{\circ} \mathrm{C}$ and incubated with anti-SIRT4 antibody $(1: 1,000$; cat. no. ab124521), anti-GAPDH antibody (1:10,000; cat. no. 5174), anti-PI3K antibody (1:1,500; cat. no. 4249), anti-Akt antibody (1:1,500; cat. no. 4691), anti-phosphorylated (p)-Akt antibody (1:500; cat. no. 4060), anti-p-inhibitor of $\kappa \mathrm{B} \alpha(\mathrm{I} \kappa \mathrm{B} \alpha)$ antibody (1:500; cat. no. 2859), anti-total (t)-IкB $\alpha$ antibody (1:1,000; cat. no. 4812), anti-p65 NF-кB antibody (1:500; cat. no. 8242) and anti-p-p65 NF- $\mathrm{KB}$ antibody (1:500; cat. no. 3033) for $2 \mathrm{~h}$ at $25^{\circ} \mathrm{C}$. Anti-SIRT4 was obtained from Abcam (Cambridge, MA, USA); all other primary antibodies were obtained from Cell Signaling Technology, Inc., (Danvers, MA, USA). Membranes were washed with TBS-0.05\% Tween 20 and incubated with horseradish peroxidase-conjugated goat anti-rabbit immunoglobulin $\mathrm{G}$ heavy and light chain secondary antibodies (1:10,000; cat. no. 7074; Cell Signaling Technology, Inc.) for $2 \mathrm{~h}$ at room temperature. This process was followed by the development of protein bands for visualization. Image-Pro Plus 6.0 software (Media Cybernetics, Silver Spring, MD, USA) was used to quantify relative protein densities. GAPDH was used as the loading control. Each experiment was replicated three times.

Cell proliferation assay. Cell proliferation was assessed using a Cell Counting Kit-8 assay (CCK-8; Beyotime Institute of 
Biotechnology, Shanghai, China), according to the manufacturer's protocols. HUVECs stably overexpressing SIRT4 and control cells were cultured in 96-well plates and treated with $50 \mu \mathrm{M}$ oxLDL for $48 \mathrm{~h}$ at $37^{\circ} \mathrm{C}$ and $5 \% \mathrm{CO}_{2}$. Then, $10 \mu \mathrm{l}$ of CCK-8 reagent was added to each well and mixed gently; cells were then incubated at $37^{\circ} \mathrm{C}$ for $4 \mathrm{~h}$. The absorbance was evaluated at $450 \mathrm{~nm}$ using a microplate reader. Survival rate $=$ optical density $(\mathrm{OD}) 450_{\mathrm{OV}-\mathrm{SIRT} 4}$ group/OD $450_{\mathrm{NC}}$ group Each experiment was repeated three times.

Apoptosis assay. Cell apoptosis was analyzed using flow cytometry. HUVECs stably overexpressing SIRT4 and $\mathrm{NC}$ cells were cultured and treated with $50 \mu \mathrm{M}$ oxLDL for $48 \mathrm{~h}$ at $37^{\circ} \mathrm{C}$ and $5 \% \mathrm{CO}_{2}$. The cells were collected and the assay was performed using a Propidium Iodide (PI)/Annexin V-fluorescein isothiocyanate (FITC) apoptosis detection kit (Nanjing Keygen Biotech Co., Ltd., Nanjing, China). Annexin V-FITC (5 $\mu \mathrm{l})$ and PI (5 $\mu \mathrm{l})$ were added, and cells were incubated at $25^{\circ} \mathrm{C}$ in the dark for $15 \mathrm{~min}$. Within $1 \mathrm{~h}$, the apoptotic cells was then assessed using a flow cytometer (BD Biosciences, San Jose, CA, USA) and data were analyzed using FlowJo version 10.07 software (FlowJo LLC, Ashland, OR, USA). The apoptotic rate was defined as the percentage of cells in the upper and lower right quadrants. Each experiment was repeated three times.

Immunofluorescence. HUVECs stably overexpressing SIRT4 and NC cells $\left(4 \times 10^{5}\right.$ cells $\left./ \mathrm{ml}\right)$ were seeded into 24 -well plates and treated with $50 \mu \mathrm{M}$ oxLDL for $48 \mathrm{~h}$ at $37^{\circ} \mathrm{C}$ and $5 \% \mathrm{CO}_{2}$. Following treatment, the cells were washed with PBS and then fixed with $4 \%$ paraformaldehyde for $15 \mathrm{~min}$ at $37^{\circ} \mathrm{C}$. The cell membranes were then permeabilized using $0.3 \%$ Triton X-100 in PBS with 0.2\% (V/V) Tween-20 (PBST) on ice for $15 \mathrm{~min}$, followed by blocking with $5 \%$ bovine serum albumin (Gibco; Thermo Fisher Scientific, Inc.) and 2.5\% fetal bovine serum (Gibco; Thermo Fisher Scientific, Inc.) in PBST and were then incubated for $2 \mathrm{~h}$ at $25^{\circ} \mathrm{C}$ with rabbit

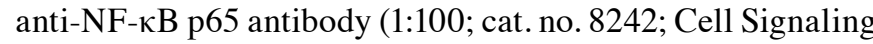
Technology, Inc.). Next, cells were washed three times with PBST and incubated with the Alexa Fluor ${ }^{\circledR}$ 594-conjugated secondary antibody (1:200; cat. no. ab150084; Abcam) for $1 \mathrm{~h}$ at room temperature. Following three washes with PBST, the cells were counterstained with DAPI (Sigma-Aldrich; Merck KGaA, Darmstadt, Germany) for $5 \mathrm{~min}$ at $25^{\circ} \mathrm{C}$. The cells were then visualized and images captured via fluorescence microscopy (magnification, x400; Leica Microsystems GmbH, Wetzlar, Germany).

Enzyme-linked immunosorbent assay (ELISA) for cytokine detection. HUVECs stably overexpressing SIRT4 and NC cells $\left(4 \times 10^{5}\right.$ cells $\left./ \mathrm{ml}\right)$ were seeded into 24 -well plates, followed by treatment with $50 \mu \mathrm{M}$ oxLDL for $48 \mathrm{~h}$ at $37^{\circ} \mathrm{C}$ and $5 \%$ $\mathrm{CO}_{2}$. Cell-free supernatants were collected and used in an assay for the detection of cytokines. The concentrations of the cytokines IL-1 $\beta$ (E-EL-H0149c), IL-6 (E-EL-H0102c) and TNF- $\alpha$ (E-EL-H0109c) were determined using commercially available ELISA kits (eBioscience; Thermo Fisher Scientific, Inc.). The entire procedure was performed according to the manufacturer's protocols. Each experiment was replicated three times.
Statistical analysis. All statistical analyses were performed using SPSS version 19.0 (IBM Corp., Armonk, NY, USA). Results are presented as the mean \pm standard deviation, and experiments were repeated in triplicate. Student's t-test was performed for comparison between two groups. Multiple groups were compared using one-way analysis of variance, followed by a least significant difference post-hoc test. $\mathrm{P}<0.05$ was considered to indicate a statistically significant difference.

\section{Results}

OxLDL reduces the expression of SIRT4 in HUVECs. In order to investigate changes in SIRT4 expression in response to oxLDL, HUVECs were treated with oxLDL at various concentrations. Western blotting results demonstrated that oxLDL treatment significantly reduced SIRT4 expression in HUVECs, compared with in control cells treated with $0 \mu \mathrm{M}$ oxLDL. Higher oxLDL concentrations resulted in lower SIRT4 expression levels indicating a dose dependent association between oxLDL concentrations and SIRT4 expression (Fig. 1A). Next, HUVECs were treated with the same oxLDL concentration $(50 \mu \mathrm{M})$ for different periods of time. Western blotting results demonstrated that oxLDL treatment significantly reduced SIRT4 expression in HUVECs compared with control cells treated with $0 \mu \mathrm{M}$ oxLDL, in a time dependent manner; longer durations of treatment resulted in lower SIRT4 expression levels (Fig. 1B).

Stably overexpressing SIRT4 in HUVECs. Cells overexpressing SIRT4 were selected by screening and treated with $50 \mu \mathrm{M}$ oxLDL for $48 \mathrm{~h}$. SIRT4 expression was determined via western blotting. SIRT4 expression levels were significantly higher in cells overexpressing SIRT4, compared with the NC group following oxLDL treatment for $48 \mathrm{~h}$ ( $\mathrm{P}<0.001$; Fig. 2). The results indicated successful overexpression of SIRT4 in HUVECs.

Overexpression of SIRT4 reverses oxLDL-induced inhibition of cell proliferation. To investigate the effect of SIRT4 overexpression in response to oxLDL on HUVEC proliferation, HUVECs overexpressing SIRT4 and NC HUVECs were treated with $50 \mu \mathrm{M}$ oxLDL for $48 \mathrm{~h}$. Cell proliferation was assessed using a CCK-8 assay. The survival rate was significantly higher in HUVECs overexpressing SIRT4, compared with NC HUVECs following oxLDL treatment (Fig. 3A). The results indicated that SIRT4 overexpression may reverse oxLDL-induced inhibition of cell proliferation. In order to explore the effects of SIRT4 overexpression in response to oxLDL on HUVEC apoptosis, HUVECs overexpressing SIRT4 and NC HUVECs were treated with $50 \mu \mathrm{M}$ oxLDL for $48 \mathrm{~h}$. Cell apoptosis was examined via flow cytometry. Following oxLDL treatment, the apoptotic rate significantly decreased in HUVECs overexpressing SIRT4 compared with in NC HUVECs (Fig. 3B and C). These results indicated that overexpression of SIRT4 may inhibit oxLDL-induced apoptosis.

Overexpression of SIRT4 inhibits the PI3K/Akt/NF- $\mathrm{BB}$ signaling pathway. In order to elucidate the role of SIRT4 in regulating the $\mathrm{PI} 3 \mathrm{~K} / \mathrm{Akt} / \mathrm{NF}-\kappa \mathrm{B}$ signaling pathway, 

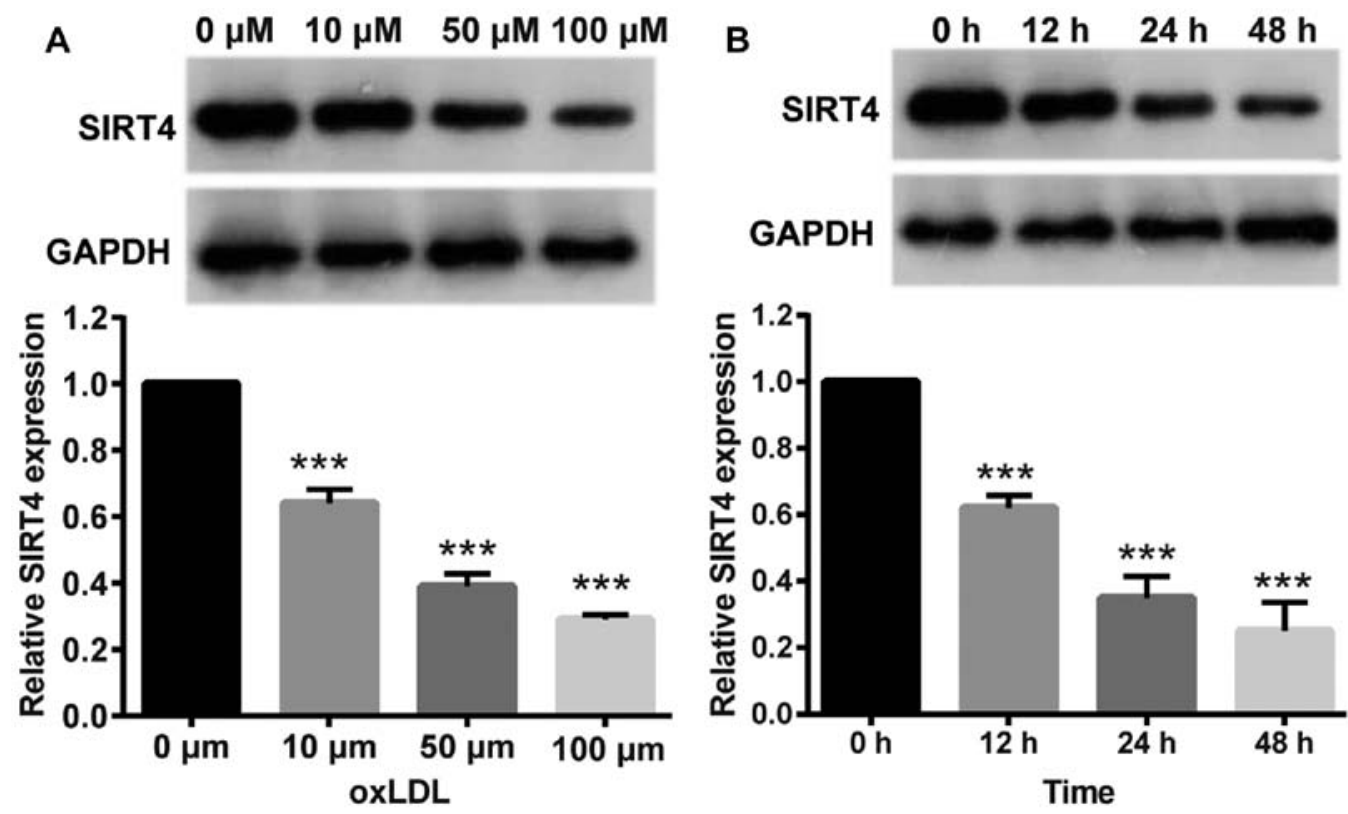

Figure 1. SIRT4 expression is altered in a dose and time-dependent manner following oxLDL treatment. Each experiment was replicated three times. (A) Relative expression of SIRT4 in response to $0,10,50$ and $100 \mu \mathrm{M}$ oxLDL. (B) Relative expression of SIRT4 in response to $50 \mu \mathrm{M}$ oxLDL for $0,12,24$ and $48 \mathrm{~h}$. ${ }^{* * *} \mathrm{P}<0.001$ vs. $0 \mu \mathrm{M}$ or 0 h. oxLDL, oxidized low density lipoprotein; SIRT4, sirtuin 4.

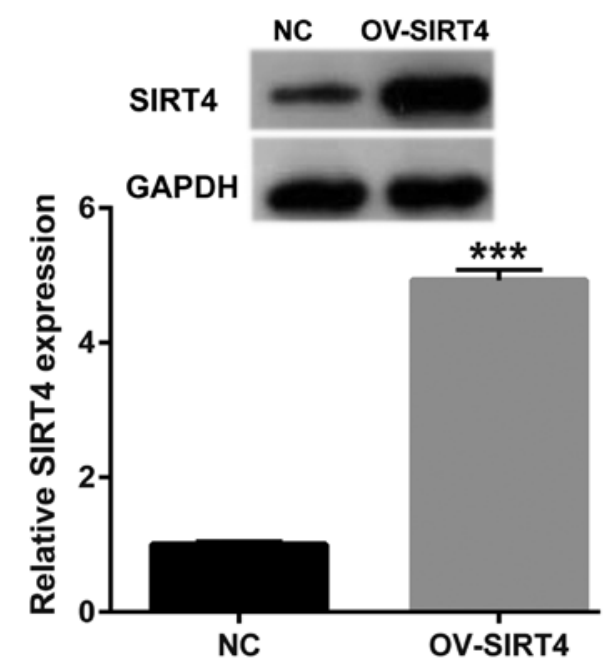

Figure 2. SIRT4 expression in overexpressed SIRT4-HUVECs and NC-HUVECs following $50 \mu \mathrm{M}$ oxLDL treatment at $48 \mathrm{~h}$. Each experiment was replicated three times. ${ }^{* * *} \mathrm{P}<0.001$ vs. NC. SIRT4, sirtuin 4; HUVECs, human umbilical vein endothelial cells; oxLDL, oxidized low density lipoprotein; NC, negative control.

HUVECs overexpressing SIRT4 and NC HUVECs were treated with $50 \mu \mathrm{M}$ oxLDL for $48 \mathrm{~h}$. Western blotting analysis was conducted to quantify protein expression associated with the PI3K/Akt/NF- $\mathrm{BB}$ signaling pathway. Compared with the NC, SIRT4 overexpression suppressed the PI3K/Akt/NF-kB pathway in HUVECs by inhibiting PI3K expression (Fig. 4A and B). It also significantly inhibited $\mathrm{p}-\mathrm{Akt}, \mathrm{p}-\mathrm{I} \kappa \mathrm{B} \alpha$ and $\mathrm{p}-\mathrm{P} 65 \mathrm{NF}-\kappa \mathrm{B}$ expression compared with the control. P65 NF- $\mathrm{kB}$ nuclear translocation was analyzed via immunofluorescence. Immunofluorescence analysis revealed that P65 NF- $\mathrm{KB}$ was largely expressed in the nucleus, while a small quantity was expressed in the cytoplasm of cells in the
oxLDL + NC treatment group. In contrast, in the oxLDL + OV-SIRT4 treatment group, $\mathrm{P} 65 \mathrm{NF}-\mathrm{\kappa B}$ was largely expressed in the cytoplasm, while a small quantity was expressed in the nucleus. These results suggest that OV-SIRT4 may inhibit P65 NF-кB nuclear translocation (Fig. 4C).

Overexpression of SIRT4 attenuates the expression of inflammatory cytokines induced by oxLDL. In order to further confirm the effect of SIRT4 overexpression on endothelial activation in response to inflammatory cytokines, HUVECs overexpressing SIRT4 and NC HUVECs were treated with $50 \mu \mathrm{M}$ oxLDL for $48 \mathrm{~h}$. The expression of IL-1 $\beta$, IL-6 and TNF- $\alpha$ were assessed using ELISA. Compared with NC HUVECs, the expression levels of IL- $1 \beta$, IL- 6 and TNF- $\alpha$ were significantly decreased in HUVECs overexpressing SIRT4 (Fig. 5). The results indicated that SIRT4 overexpression attenuated the expression levels of IL- $1 \beta$, IL- 6 and TNF- $\alpha$ induced at the protein level by oxLDL.

\section{Discussion}

Previous studies have indicated that endothelial dysfunction may serve a vital role during the initial stages of atherosclerosis $(6,24)$. Although much progress has been made in studies of pathophysiology and clinical aspects of atherosclerosis, the detailed mechanism underlying this disease remains to be elucidated $(25,26)$. OxLDL reportedly induces endothelial cell injury and dysfunction (27). In the present study, HUVECs were stimulated by oxLDL to mimic the conditions of damage and inflammation of endothelial cells. The results demonstrated that oxLDL may reduce SIRT4 expression in HUVECs in a dose- and time-dependent manner. SIRT4 is a member of the sirtuin protein family which is able to improve vascular smooth muscle and endothelial cell injury induced by lipid deposition, oxidative stress and inflammation, thus opposing 

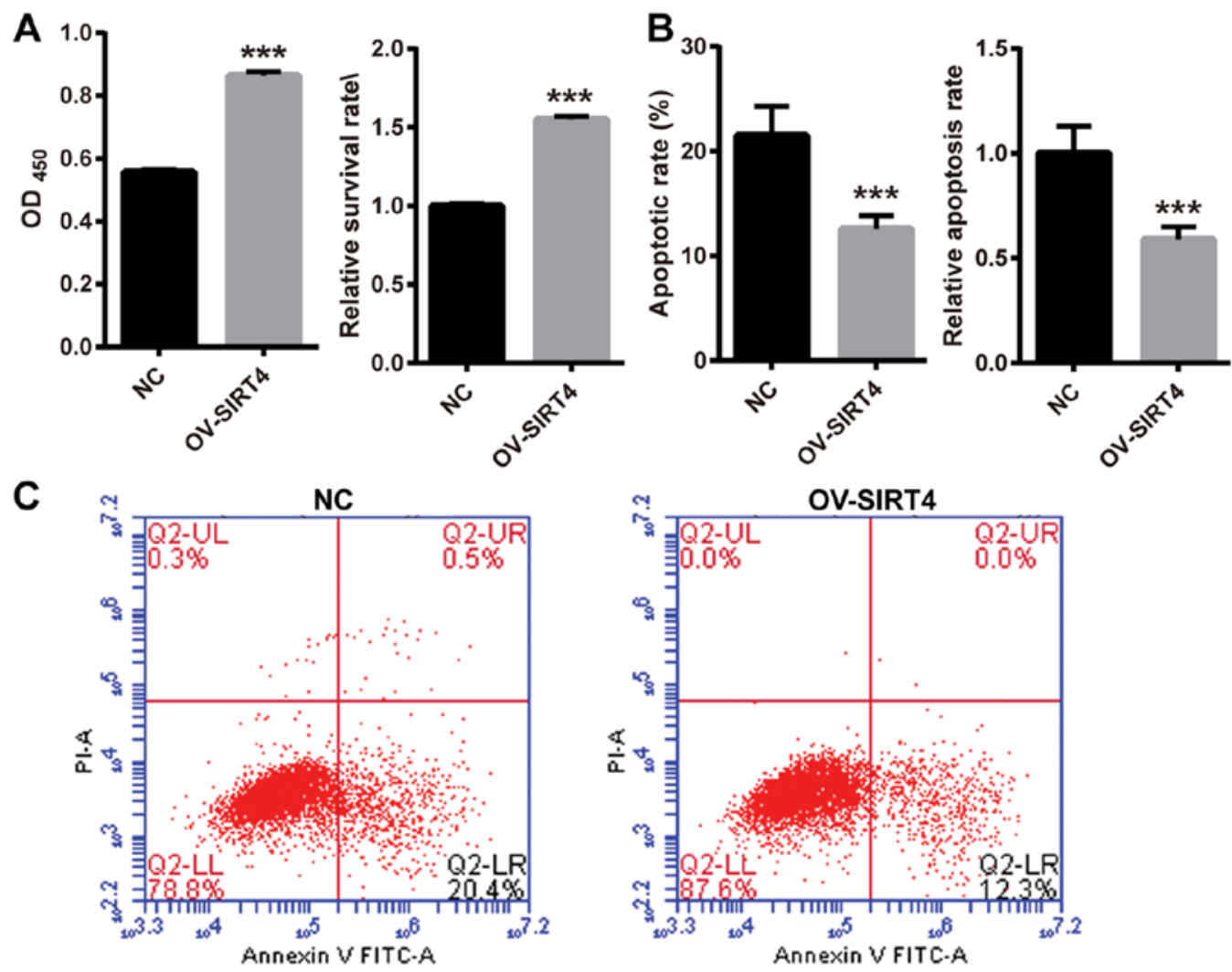

Figure 3. Effect of SIRT4 on HUVEC proliferation and apoptosis following $50 \mu \mathrm{M}$ oxLDL treatment. Each experiment was replicated three times. (A) Cell proliferation was analyzed using CCK8 reagent following $50 \mu \mathrm{M}$ oxLDL treatment at $48 \mathrm{~h}$ in overexpressed SIRT4-HUVECs and NC-HUVECs. (B and C) Cell apoptosis was analyzed using flow cytometry following $50 \mu \mathrm{M}$ oxLDL treatment at $48 \mathrm{~h}$ in overexpressed SIRT4-HUVECs and NC-HUVECs. Upper right and lower right quadrants contained the apoptotic cells $\left({ }^{* * *} \mathrm{P}<0.001\right.$ vs. NC). CCK-8, Cell Counting Kit 8 ; FITC, fluorescein isothiocyanate; HUVECs, human umbilical vein endothelial cells; NC, negative control; oxLDL, oxidized low density lipoprotein; PI, propidium iodide; SIRT4, sirtuin 4.

A

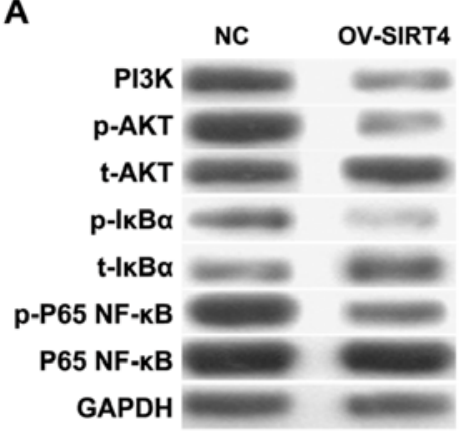

C

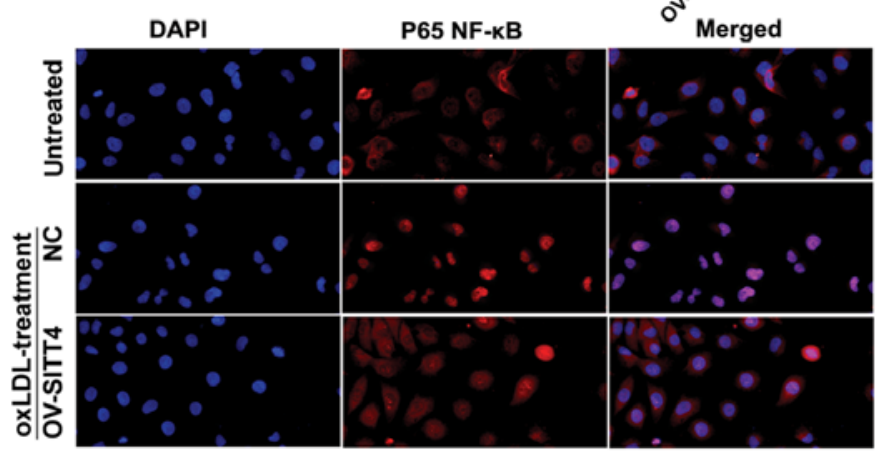

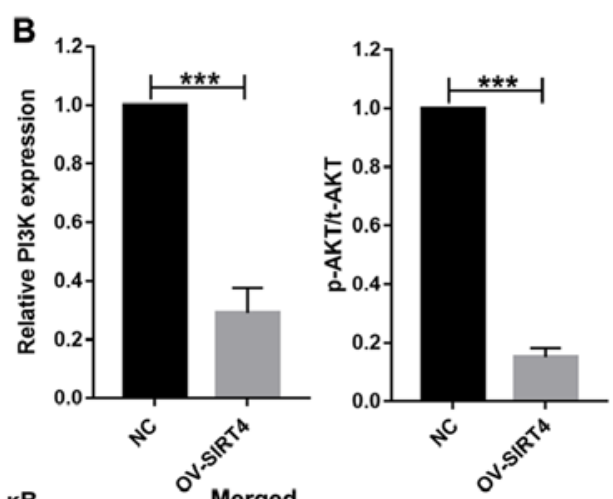
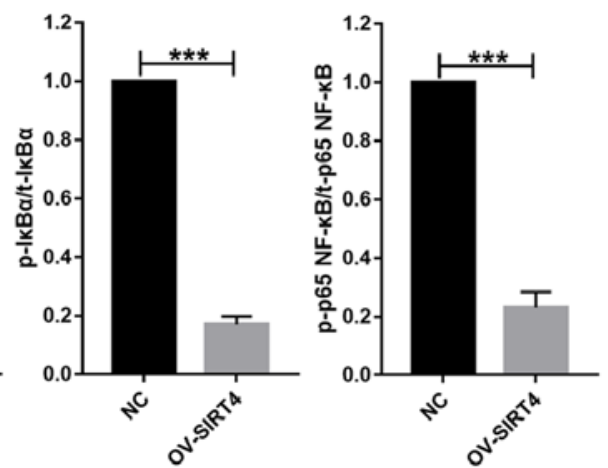

Figure 4. PI3K/Akt/NF- $\mathrm{B}$ signaling pathway, activated by oxLDL, was suppressed by SIRT4 overexpression in HUVECs. (A and B) Expression of PI3K, p-Akt, t-Akt, p-I $\kappa \mathrm{B} \alpha, \mathrm{t}-\mathrm{I} \kappa \mathrm{B} \alpha, \mathrm{p}-\mathrm{p} 65 \mathrm{NF}-\kappa \mathrm{B}, \mathrm{p} 65 \mathrm{NF}-\kappa \mathrm{B}$ proteins were detected by western blotting following $50 \mu \mathrm{M}$ oxLDL treatment at $48 \mathrm{~h}$ in overexpressed SIRT4-HUVECs and NC-HUVECs. Each experiment was replicated three times ${ }^{* * *} \mathrm{P}<0.001$ vs. NC). (C) P65 NF- $\kappa \mathrm{B}$ nuclear translocation was examined via immunofluorescence in overexpressed SIRT4-HUVECs, NC-HUVECs and HUVECs (magnification, x400). HUVECs, human umbilical vein endothelial cells; I $\mathrm{B} \alpha$, inhibitor of $\kappa \mathrm{B} \alpha$; NF, nuclear factor; NF, nuclear factor, NC, negative control; oxLDL, oxidized low density lipoprotein; PI3K, phosphoinositide 3-kinase; Akt, protein kinase B; SIRT4, sirtuin 4; p-, phosphorylated; t-, total. 

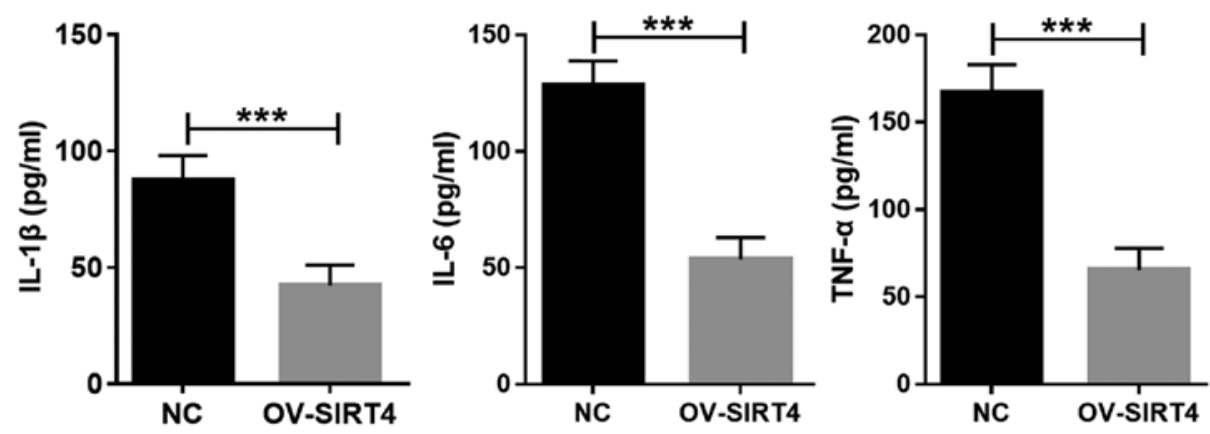

Figure 5. Overexpression of SIRT4 attenuates the expression of IL-1 $\beta$, IL-6 and TNF- $\alpha$ induced by oxLDL. Expression levels of IL-1 $\beta$, IL- 6 and TNF- $\alpha$ following $50 \mu \mathrm{M}$ oxLDL treatment at $48 \mathrm{~h}$ in overexpressed SIRT4-HUVECs and NC-HUVECs. Each experiment was replicated three times. ${ }^{* * *} \mathrm{P}<0.001$ vs. NC. HUVECs, human umbilical vein endothelial cells; IL, interleukin; TNF, tumor necrosis factor; NC, negative control; oxLDL, oxidized low density lipoprotein; SIRT4, sirtuin 4.

atherogenesis (28). In a previous study, it was demonstrated that LPS treatment significantly reduced SIRT4 expression at the mRNA and protein levels in a dose-dependent manner (16). In the present study, it was demonstrated that oxLDL treatment inhibited SIRT4 expression in a dose-dependent manner in HUVECs, suggesting that SIRT4 may be critically involved in oxLD L-induced endothelial dysfunction. In diabetic nephropathy, SIRT4 overexpression increased mouse podocyte cell proliferation and suppressed apoptosis, which was accompanied with an increase in mitochondrial membrane potential and decreased production of reactive oxygen species (18). The results of the present study demonstrated that overexpression of SIRT4 may reverse oxLDL-induced cell proliferation inhibition and rescue oxLDL-induced apoptosis. These results indicated that SIRT4 overexpression may enhance the survival of HUVECs in an oxLDL-induced inflammatory environment.

It has been reported that the PI3K/Akt pathway may be implicated in the regulation of a number of inflammatory diseases, including atherosclerosis (29). In addition, it was previously demonstrated that activation of the PI3K/Akt pathway was also involved in the activation of NF- $\mathrm{KB}$ via the phosphorylation of $\mathrm{I} \kappa \mathrm{B} \alpha$ protein (30). The NF- $\kappa \mathrm{B}$ complex is localized and inactivated in the cytoplasm by binding to the inhibitory protein $\mathrm{I} \kappa \mathrm{B}$ (31). Phosphorylation of $I \kappa B \alpha$ protein results in the dissociation of $I \kappa B \alpha$ from $N F-\kappa B$, allowing NF- $\kappa B$ to freely migrate into the nucleus and activate transcription (32). The current study identified that SIRT4 overexpression suppressed PI3K, p-Akt, p-IкB $\alpha$ and p-P65 NF- $\kappa \mathrm{B}$ expression. Furthermore, immunofluorescence analysis revealed that $\mathrm{p} 65 \mathrm{NF}-\kappa \mathrm{B}$, which is localized in the nucleus, was significantly decreased in HUVECs with SIRT4-overexpression. These results suggested that SIRT4

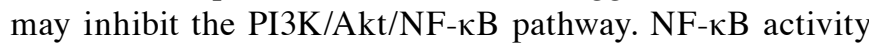
has been demonstrated to be essential for the production of pro-inflammatory cytokines in atherosclerosis. Inflammation contributes to AS development from plaque onset to plaque rupture (33). Inflammatory cytokines promote the recruitment of monocytes to endothelial cells and induce to foam cell formation (34). A previous study demonstrated that SIRT4 suppressed NF- $\mathrm{kB}$ nuclear translocation, and inhibited IL-1 $\beta$, IL-6 and IL-8 expression in HUVECs (19). The findings of the present study demonstrated that SIRT4 overexpression significantly decreased the levels of IL- $1 \beta$, IL- 6 and TNF- $\alpha$ induced by oxLDL. The results of the present study indicated that SIRT4 overexpression attenuated the expression of IL-1 $\beta$, IL- 6 and TNF- $\alpha$ induced by oxLDL by suppressing the $\mathrm{PI} 3 \mathrm{~K} / \mathrm{Akt} / \mathrm{NF}-\mathrm{KB}$ pathway, which improves the inflammatory environment induced by oxLDL and enhances the survival of HUVECs.

The present study involved several limitations. First, all experiments were performed using one cell line and, therefore, it is possible that the conclusions are specific to this cell line only and may not apply to atherosclerosis per se. Thus, further validation of these findings using more cell and animal models are required in subsequent studies. Additionally, the potential pathways that mediate the effects of oxLDL on SIRT4 and the effects of SIRT4 on the PI3K/Akt pathway remain to be elucidated. Whether SIRT4 attenuates oxLDL-induced HUVEC apoptosis via the PI3K/Akt/NF-kB signaling pathway requires further investigation.

In conclusion, the present study provided notable evidence suggesting that SIRT4 overexpression enhanced HUVEC survival, suppressed the PI3K/Akt/NF- $\kappa B$ signaling pathway and inhibited inflammatory cytokine expression induced by oxLDL. These results may serve as a theoretical basis to provide novel insight into the treatment of atherosclerosis.

\section{Acknowledgements}

Not applicable.

\section{Funding}

This study was supported by the grant from Jiangxi Province Science Foundation for Youths (grant no. 20161BAB215257) and the Science and Technology Program by the Health and Family Planning Commission of Jiangxi Province (grant no. 20162304).

\section{Availability of data and materials}

The datasets used and/or analyzed during the present study are available from the corresponding author on reasonable request.

\section{Authors' contributions}

YT, SY and HL conceived and designed the present study. MC, YW and JX developed the methodology. YT, SY, MC, 
YW and JX completed the experiment and collected the data. YT, SY and HL analyzed and interpreted the data. YT and SY drafted the manuscript. All authors read and approved the final manuscript.

\section{Ethics approval and consent to participate}

Not applicable.

\section{Patient consent for publication}

Not applicable.

\section{Competing interests}

The authors declare that they have no competing interests.

\section{References}

1. Heuslein JL, Meisner JK, Li X, Song J, Vincentelli H Leiphart RJ, Ames EG, Blackman BR, Blackman BR and Price RJ: Mechanisms of amplified arteriogenesis in collateral artery segments exposed to reversed flow direction. Arterioscler Thromb Vasc Biol 35: 2354-2365, 2015.

2. Tousoulis D, Oikonomou E, Economou EK, Crea F and Kaski JC: Inflammatory cytokines in atherosclerosis: Current therapeutic approaches. Eur Heart J 37: 1723-1732, 2016.

3. Park KH and Park WJ: Endothelial dysfunction: Clinical implications in cardiovascular disease and therapeutic approaches. J Korean Med Sci 30: 1213-1225, 2015.

4. Tuttolomondo A, Di Raimondo D, Pecoraro R, Arnao V, Pinto A and Licata G: Atherosclerosis as an inflammatory disease. Curr Pharm Des 18: 4266-4288, 2012.

5. Chistiakov DA, Melnichenko AA, Grechko AV, Myasoedova VA and Orekhov AN: Potential of anti-inflammatory agents for treatment of atherosclerosis. Exp Mol Pathol 104: 114-124, 2018.

6. Ren K, Lu YJ, Mo ZC, -Liu X, Tang ZL, Jiang Y, Peng XS, Li L, Zhang QH and Yi GH: ApoA-I/SR-BI modulates S1P/S1PR2-mediated inflammation through the PI3K/Akt signaling pathway in HUVECs. J Physiol Biochem 73: 287-296, 2017.

7. Pirinen E, Lo Sasso G and Auwerx J: Mitochondrial sirtuins and metabolic homeostasis. Best Pract Res Clin Endocrinol Metab 26: 759-770, 2012.

8. Kane AE and Sinclair DA: Sirtuins and NAD(+) in the development and treatment of metabolic and cardiovascular diseases. Circ Res 123: 868-885, 2018.

9. Zheng H, Fu Y, Huang Y, Zheng X, Yu W and Wang W: mTOR signaling promotes foam cell formation and inhibits foam cell egress through suppressing the SIRT1 signaling pathway. Mol Med Rep 16: 3315-3323, 2017.

10. D'Onofrio N, Servillo L and Balestrieri ML: SIRT1 and SIRT6 signaling pathways in cardiovascular disease protection. Antioxid Redox Signal 28: 711-732, 2018.

11. Zhang B, Ma Y and Xiang C: SIRT2 decreases atherosclerotic plaque formation in low-density lipoprotein receptor-deficient mice by modulating macrophage polarization. Biomed Pharmacother 97: 1238-1242, 2018.

12. Haigis MC, Mostoslavsky R, Haigis KM, Fahie K, Christodoulou DC, Murphy AJ, Valenzuela DM, Yancopoulos GD, Karow M, Blander G, et al: SIRT4 inhibits glutamate dehydrogenase and opposes the effects of calorie restriction in pancreatic beta cells. Cell 126: 941-954, 2006.

13. Komlos D, Mann KD, Zhuo Y, Ricupero CL, Hart RP, Liu AY and Firestein BL: Glutamate dehydrogenase 1 and SIRT4 regulate glial development. Glia 61: 394-408, 2013.

14. Michishita E, Park JY, Burneskis JM, Barrett JC and Horikawa I Evolutionarily conserved and nonconserved cellular localizations and functions of human SIRT proteins. Mol Biol Cell 16 4623-4635, 2005.
15. Laurent G, de Boer VC, Finley LW, Sweeney M, Lu H, Schug TT, Cen Y, Jeong SM, Li X, Sauve AA and Haigis MC: SIRT4 represses peroxisome proliferator-activated receptor $\alpha$ activity to suppress hepatic fat oxidation. Mol Cell Biol 33: 4552-4561, 2013.

16. Laurent G,German NJ,Saha AK, de Boer VC,Davies M,Koves TR, Dephoure N, Fischer F, Boanca G, Vaitheesvaran B, et al: SIRT4 coordinates the balance between lipid synthesis and catabolism by repressing malonyl CoA decarboxylase. Mol Cell 50: 686-698, 2013.

17. Qiu Y, Lai H, Huang Y, Hong L, Wang $\mathrm{H}$ and Tao Y: Effect of shear force on SIRT4 in LPS-injured human umbilical vein endothelial cells. Int J Clin Exp Pathol 9: 4921-4930, 2016.

18. Miyo M, Yamamoto H, Konno M, Colvin H, Nishida N, Koseki J, Kawamoto K, Ogawa H, Hamabe A, Uemura M, et al: Tumour-suppressive function of SIRT4 in human colorectal cancer. Br J Cancer 113: 492-499, 2015.

19. Shi JX, Wang QJ, Li H and Huang Q: SIRT4 overexpression protects against diabetic nephropathy by inhibiting podocyte apoptosis. Exp Ther Med 13: 342-348, 2017

20. Tao Y, Huang C, Huang Y, Hong L, Wang H, Zhou Z and Qiu Y: SIRT4 suppresses inflammatory responses in human umbilical vein endothelial cells. Cardiovasc Toxicol 15: 217-223, 2015.

21. Hasanally D, Edel A, Chaudhary R and Ravandi A: Identification of oxidized phosphatidylinositols present in OxLDL and human atherosclerotic plaque. Lipids 52: 11-26, 2017.

22. Kang Q, Liu W, Liu H and Zhou M: Effect of compound chuanxiong capsule on inflammatory reaction and PI3K/Akt/NF- $\kappa \mathrm{B}$ signaling pathway in atherosclerosis. Evid Based Complement Alternat Med 2015: 584596, 2015.

23. Schlegel N, Leweke R, Meir M, Germer CT and Waschke J: Role of NF- $\kappa \mathrm{B}$ activation in LPS-induced endothelial barrier breakdown. Histochem Cell Biol 138: 627-641, 2012.

24. Liu Z, Wang J, Huang X, Li Z and Liu P: Deletion of sirtuin 6 accelerates endothelial dysfunction and atherosclerosis in apolipoprotein E-deficient mice. Transl Res 172: 18-29.e2, 2016.

25. Jackson SW, Scharping NE, Jacobs HM, Wang S, Chait A and Rawlings DJ: Cutting Edge: BAFF overexpression reduces atherosclerosis via TACI-Dependent B cell activation. J Immunol 197: 4529-4534, 2016

26. Min J, Weitian Z, Peng C, Yan P, Bo Z, Yan W, Yun B and Xukai W: Correlation between insulin-induced estrogen receptor methylation and atherosclerosis. Cardiovasc Diabetol 15: 156, 2016.

27. Di Pietro N, Formoso G and Pandolfi A: Physiology and pathophysiology of oxLDL uptake by vascular wall cells in atherosclerosis. Vascul Pharmacol 84: 1-7, 2016.

28. Sosnowska B, Mazidi M, Penson P, Gluba-Brzózka A, Rysz J and Banach M: The sirtuin family members SIRT1, SIRT3 and SIRT6: Their role in vascular biology and atherogenesis. Atherosclerosis 265: 275-282, 2017.

29. Garat CV, Crossno JT Jr, Sullivan TM, Reusch JE and Klemm DJ: Inhibition of phosphatidylinositol 3-kinase/Akt signaling attenuates hypoxia-induced pulmonary artery remodeling and suppresses CREB depletion in arterial smooth muscle cells. J Cardiovasc Pharmacol 62: 539-548, 2013.

30. Shen T, Yang Z, Cheng X, Xiao Y, Yu K, Cai X, Xia C and Li Y: CXCL8 induces epithelial-mesenchymal transition in colon cancer cells via the PI3K/Akt/NF- $\kappa \mathrm{B}$ signaling pathway. Oncol Rep 37: 2095-2100, 2017.

31. Napetschnig $\mathbf{J}$ and $\mathrm{Wu} \mathrm{H}$ : Molecular basis of NF-kB signaling. Annu Rev Biophys 42: 443-468, 2013.

32. Hayden MS and Ghosh S: NF- $\kappa \mathrm{B}$, the first quarter-century: remarkable progress and outstanding questions. Genes Dev 26: 203-234, 2012

33. Onat D, Brillon D, Colombo PC and Schmidt AM: Human vascular endothelial cells: A model system for studying vascular inflammation in diabetes and atherosclerosis. Curr Diab Rep 11: 193-202, 2011.

34. Zernecke A and Weber C: Inflammatory mediators in atherosclerotic vascular disease. Basic Res Cardiol 100: 93-101, 2005. 